\title{
Search for new physics in CP violation with beauty and charm decays at LHCb
}

Matteo Bartolini ${ }^{* \dagger}$

University and INFN Genova

E-mail: matteo.bartolini@cern.ch

\begin{abstract}
$\mathrm{LHCb}$ is one of the four big experiments operating at LHC and it's mainly dedicated to measurements of $C P$ violation and to the search for new physics in the decays of rare hadrons containing heavy quarks. The LHCb collaboration has recently published a result which shows for the first time a compelling $5.3 \sigma$ evidence of $C P$ violation in the two-body meson $D^{0} \rightarrow K^{+} K^{-}$and $D^{0} \rightarrow \pi^{+} \pi^{-}$decays.

$C P$ violation in the Cabibbo-suppressed decays $D_{s}^{+} \rightarrow K_{S}^{0} \pi^{+}, D^{+} \rightarrow K_{S}^{0} K^{+}, D^{+} \rightarrow \phi \pi^{+}$is expected to be small $\left(\sim 10^{-3}\right)$ due to interference between tree and penguin diagrams and thus sensible to contributions Beyond Standard Model (BSM). The last measurement by LHCb of direct $C P$ asymmetries in the aforementioned decay channels shows consistency with the hypothesis of $C P$ conservation.

In the $B_{s}$ sector the $C P$ violating phase $\phi_{s}$ arises from the interference between direct decay and decay after mixing and may also be sensitive to BSM contributions. The last two published measurements of $\phi_{s}$ using the $B_{s} \rightarrow J / \psi K^{+} K^{-}$and $B_{s} \rightarrow J / \psi \pi^{+} \pi^{-}$decay channels show a combined $1.6 \sigma$ hint of $C P$ violation.
\end{abstract}

XXVII International Workshop on Deep-Inelastic Scattering and Related Subjects - DIS2019 8-12 April, 2019

Torino, Italy

* Speaker.

${ }^{\dagger}$ On behalf of the LHCb Collaboration. 


\section{Introduction}

LHCb is a large experiment collecting data at the LHC and one of its primary goal is to look for indirect evidence of new physics in $C P$ violation as this may help explain the absence of antimatter in the Universe today.

The LHCb detector is a single-arm forward spectrometer designed for the study of particles containing $b$ or $c$ quarks in the pseudorapidity range $2<\eta<5$ [1]. The detector includes a high-precision vertex detector (VELO)[2] surrounding the $p p$ interaction region which is able to measure the impact parameter (IP) of a track with resolutions down to $15 \mu \mathrm{m}$. Final state particles like pions, kaons and protons are identified in a wide momentum range via two Ring Imaging Cherenkov detectors $(\mathrm{RICH})[3]$.

\section{CP violation in the charm sector}

The size of $C P$ violation in charm decays is expected to be small in the SM, with asymmetries of the order of $10^{-3}-10^{-4}[4]$, but contributions of BSM virtual particles may alter its size with respect to the SM expectations[5].

The LHCb collaboration reported the search for $C P$ violation in $D^{0} \rightarrow K^{-} K^{+}$and $D^{0} \rightarrow \pi^{-} \pi^{+}$ decays using $p p$ collision data corresponding to an integrated luminosity of $5.9 \mathrm{fb}^{-1}$ collected at a centre-of-mass energy of $13 \mathrm{TeV}$, announcing the first observation of a time-integrated $C P$ asymmetry in the charm quark sector[6].

The time-dependent $C P$ asymmetry $A_{C P}(f ; t)$ between states produced as $D^{0}$ or $\bar{D}^{0}$ decaying to a $C P$ eigenstate $f$ at time $t$ is defined as

$$
A_{C P}(f ; t) \equiv \frac{\Gamma\left(D^{0}(t) \rightarrow f\right)-\Gamma\left(\bar{D}^{0}(t) \rightarrow f\right)}{\Gamma\left(D^{0}(t) \rightarrow f\right)+\Gamma\left(\bar{D}^{0}(t) \rightarrow f\right)} .
$$

The flavor of the charm meson is inferred from the charge of the pion in prompt $D^{*}(2010)^{+} \rightarrow$ $D^{0} \pi^{+}$decays or from the charge of the muon in $\bar{B} \rightarrow D^{0} \mu^{-} \bar{v}_{\mu} X$ decays.

The time-integrated raw asymmetries are determined by fits to the $m\left(D^{0} \pi^{+}\right)$and $m\left(D^{0}\right)$ distributions for the $\pi$-tagged and $\mu$-tagged candidates respectively as shown in Fig. 1. These raw asymmetries include contributions from different reconstruction efficiencies between positive and negative tagging pions and muons as well as production asymmetries of $D^{*}$ mesons and $b$ hadrons

$$
\begin{gathered}
A_{\text {raw }}^{\pi-\text { tagged }}(f) \approx A_{C P}(f)+A_{D}(\pi)+A_{P}\left(D^{*}\right), \\
A_{\text {raw }}^{\mu-\text { tagged }}(f) \approx A_{C P}(f)+A_{D}(\mu)+A_{P}(B) .
\end{gathered}
$$

The values of $A_{D}(\pi)$ and $A_{P}\left(D^{*}\right)$, as well as those of $A_{D}(\mu)$ and $A_{P}(B)$, are independent of the final state $f$ and cancel in the difference if the kinematics are equalised, resulting in $\Delta A_{C P} \equiv$ $A_{C P}\left(K^{-} K^{+}\right)-A_{C P}\left(\pi^{+} \pi^{-}\right) \equiv A_{\text {raw }}\left(K^{-} K^{+}\right)-A_{\text {raw }}\left(\pi^{-} \pi^{+}\right)$.

The distributions of the transverse momentum, azimuthal angle and pseudorapidity of $D^{*}$ and $D^{0}$ mesons are reweighted such that they match for the $K^{+} K^{-}$and $\pi^{+} \pi^{-}$decay modes to ensure perfect cancellation of the spurious asymmetries.

The difference between the $C P$ asymmetries in $D^{0} \rightarrow K^{-} K^{+}$and $D^{0} \rightarrow \pi^{-} \pi^{+}$decays is measured 
to be $\Delta A_{C P}=[-18.2 \pm 3.2$ (stat.) \pm 0.9 (syst.) $] \times 10^{-4}$ for $\pi$-tagged and $\Delta A_{C P}=[-9 \pm 8$ (stat.) \pm 5 (syst.) $] \times 10^{-4}$ for $\mu$-tagged $D^{0}$ mesons. Combining these with previous LHCb results $[7,8]$ leads to

$$
\Delta A_{C P}=(-15.4 \pm 2.9) \times 10^{-4}
$$

The significance of the deviation from zero corresponds to 5.3 standard deviations.
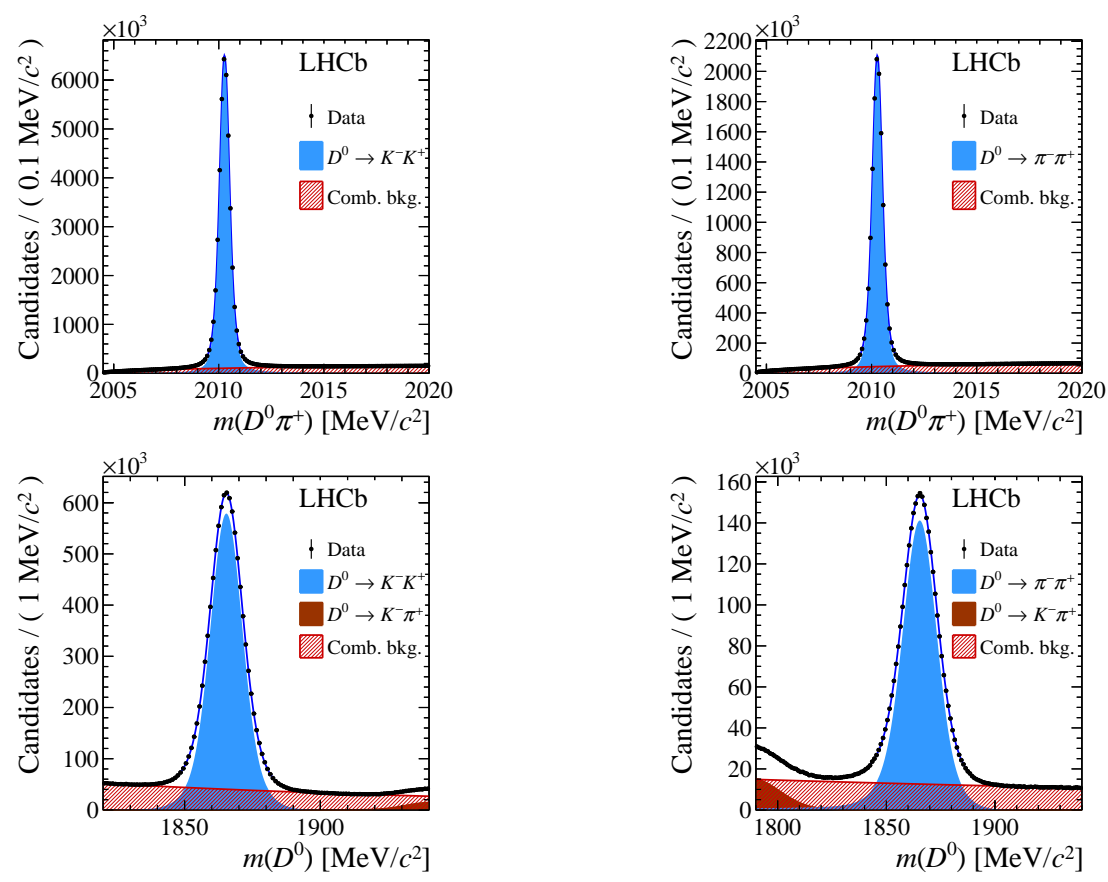

Figure 1: Mass distributions of selected (top) $\pi$-tagged and (bottom) $\mu$-tagged candidates for (left) $K^{+} K^{-}$ and (right) $\pi^{+} \pi^{-}$final states of the $D^{0}$-meson decays, with fit projections overlaid.

The LHCb collaboration has recently released results on the search for $C P$ violation in the Cabibbo-suppressed $D_{S}^{+} \rightarrow K_{S}^{0} \pi^{+}, D^{+} \rightarrow K_{S}^{0} K^{+}, D^{+} \rightarrow \phi \pi^{+}$decays using data from $p p$ collision corresponding to an integrated luminosity of $3.8 \mathrm{fb}^{-1}$ collected at a centre-of-mass energy of 13 $\mathrm{TeV}$ [9]. The raw $C P$ asymmetry of a prompt $D_{(s)}^{+}$meson decaying to the final state $f^{+}$is given by the difference in the observed yields $N$ and it is determined by fits to the mass distributions shown in Fig. 2

$$
A_{\text {raw }}\left(D_{(s)}^{+} \rightarrow f^{+}\right) \equiv \frac{N\left(D_{(s)}^{+} \rightarrow f^{+}\right)-N\left(D_{(s)}^{+} \rightarrow f^{+}\right)}{N\left(D_{(s)}^{+} \rightarrow f^{+}\right)+N\left(D_{(s)}^{+} \rightarrow f^{+}\right)} .
$$

The raw asymmetry can be approximated as

$$
A_{\text {raw }}\left(D_{(s)}^{+} \rightarrow f^{+}\right) \approx A_{C P}\left(D_{(s)}^{+} \rightarrow f^{+}\right)+A_{P}\left(D_{(s)}^{+}\right)+A_{D}\left(f^{+}\right),
$$

where $A_{P}\left(D_{(s)}^{+}\right)$is the $D_{(s)}^{+}$production asymmetry and $A_{D}\left(f^{+}\right)$is the detection asymmetry for the final state $f^{+}$. The production and detection asymmetries are canceled using the $D^{+} \rightarrow K_{S}^{0} \pi^{+}$, $D_{s}^{+} \rightarrow K_{S}^{0} K^{+}, D_{s}^{+} \rightarrow \phi \pi^{+}$decays, which proceed through the Cabibbo-favored $c \rightarrow s \bar{d} u$ transition. These high-yield samples of kinematically and topologically similar decays are expected to have 
$C P$ asymmetries that are negligible compared to the Cabibbo-suppressed modes.

The measured $C P$ asymmetries are:

$$
\begin{gathered}
A_{C P}\left(D_{s}^{+} \rightarrow K_{S}^{0} \pi^{+}\right)=[1.3 \pm 1.9(\text { stat. }) \pm 0.5(\text { syst. })] \times 10^{-3}, \\
A_{C P}\left(D^{+} \rightarrow K_{S}^{0} K^{+}\right)=[-0.09 \pm 0.65(\text { stat. }) \pm 0.48(\text { syst. })] \times 10^{-3}, \\
\left.A_{C P}\left(D^{+} \rightarrow \phi \pi^{+}\right)=[0.05 \pm 0.42 \text { (stat. }) \pm 0.29(\text { syst. })\right] \times 10^{-3} .
\end{gathered}
$$

They are the most precise measurements of these quantities to date and are consistent with the hypothesis of $C P$ conservation.
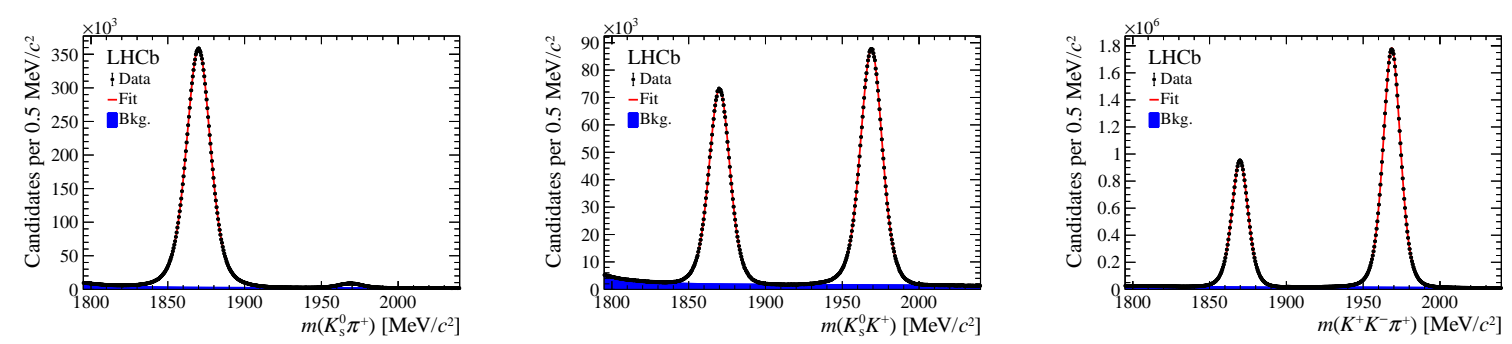

Figure 2: Mass distribution of the selected $D_{(s)}^{+} \rightarrow K_{S}^{0} \pi^{+}$(left), $D_{(s)}^{+} \rightarrow K_{S}^{0} K^{+}$(middle), $D_{(s)}^{+} \rightarrow \phi \pi^{+}$(right) candidates with fit projection overlaid.

\section{CP violation in the beauty sector}

In certain $B_{s}^{0}$ decays to $C P$ eigenstates the $C P$-violating phase $\phi_{s}$ can be expressed in terms of CKM matrix elements as $-2 \arg \left[-V_{t s} V_{t b}^{*} / V_{c s} V_{c b}^{*}\right]$. Global fits to experimental data, assuming unitarity of the CKM matrix, give a precise prediction of this value [10].

LHCb has recently released a new measurement of $\phi_{s}$ using data from $p p$ collisions corresponding to an integrated luminosity of $1.9 \mathrm{fb}^{-1}$ and collected at a centre-of-mass energy of $13 \mathrm{TeV}$ analyzing the decays $B_{s}^{0} \rightarrow J / \psi \pi^{+} \pi^{-}[11]$ and $B_{s}^{0} \rightarrow J / \psi K^{+} K^{-}[12]$. The mass distributions of the selected $B_{s}^{0}$ candidates are shown in Fig. 3.

The decay rates for $B_{s}^{0}$ and $\bar{B}_{s}^{0}$ into the final state is written as

$$
\left(\bar{\Gamma}(t) \propto e^{-\Gamma_{s} t}\left(\frac{A^{2}+\bar{A}^{2}}{2} \cosh \frac{\Delta \Gamma_{s} t}{2} \pm \frac{A^{2}-\bar{A}^{2}}{2} \cos \left(\Delta m_{s} t\right)-\operatorname{Re}\left(A^{*} \bar{A}\right) \sinh \frac{\Delta \Gamma_{s} t}{2} \mp \operatorname{Im}\left(A^{*} \bar{A}\right) \sin \left(\Delta m_{s} t\right)\right),\right.
$$

where $\Gamma_{s}=\frac{\Gamma_{L}+\Gamma_{H}}{2}, \Delta \Gamma_{s}=\Gamma_{L}-\Gamma_{H}, \Delta m_{s}=m_{H}-m_{L}$.

Because of the spin $1 J / \psi$ meson in the final state, the tree possible polarizations of the $J / \psi$ cause the decay amplitudes $A\left(B_{s}^{0} \rightarrow f\right)=\sum A_{i}$ and $\bar{A}\left(\bar{B}_{s}^{0} \rightarrow f\right)=\sum \eta_{i}\left|\lambda_{i}\right| e^{-i \phi_{s}^{i}} A_{i}$ to be and admixture of $C P$-even $\left(\eta_{i}=1\right)$ and $C P$-odd $\left(\eta_{i}=-1\right)$ eigenstates due to angular momentum conservation. The amplitudes $A_{i}$ are functions of the $h^{+} h^{-}$invariant mass and three angular variables defined in the helicity base [13], $\phi_{s}^{i}$ is the phase difference while $\lambda_{i}=\frac{q \bar{A}_{i}}{p A_{i}}$ relates $C P$ violation in the interference between mixing and decay associated with the polarization state $i$ for each resonance in the final state. The resonant structure of these two decays has already been studied in details using $p p$ collisions at 7 and $8 \mathrm{TeV}[14,15]$ and it is shown in Fig. 4 . 

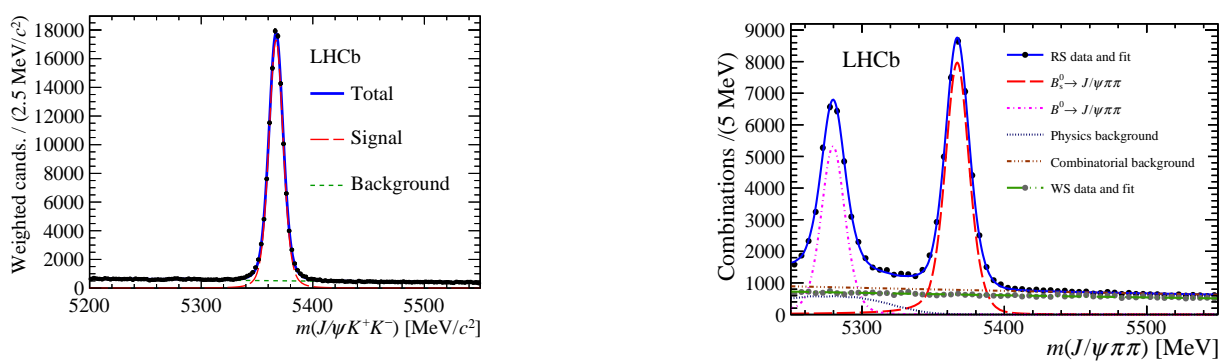

Figure 3: Invariant mass of the $B_{s}^{0} \rightarrow J / \psi K^{+} K^{-}$(left) and $B_{s}^{0} \rightarrow J / \psi \pi^{+} \pi^{-}$(right) decays with fits overlaid.
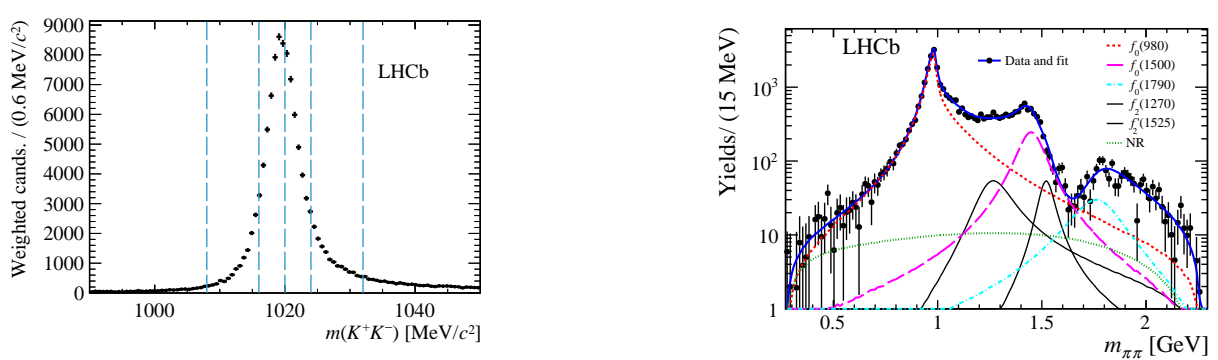

Figure 4: Distribution of the $K^{+} K^{-}$(left) and $\pi^{+} \pi^{-}$(right) invariant mass of the two $B_{s}^{0}$ decays. The former was divided in 6 bins in the analysis whereas the latter was modeled with 5 resonances and a NR amplitude .

The multi-dimensional fits to the distribution defined in (3.1), including acceptance corrections, angular efficiencies and calibration of the flavour-tagging algorithm, provides the $C P$-violating parameters $\phi_{s}$ and $|\lambda|$. In the fit $\phi_{s}^{i}$ and $\left|\lambda^{i}\right|$ are assumed to be the same for all polarization states i whereas $|q / p|$ is taken to be equal to 1 according to the last measurement by $\mathrm{LHCb}[16]$.

The fits results are $\phi_{s}=-0.083 \pm 0.041 \pm 0.006 \mathrm{rad}$ and $|\lambda|=1.006 \pm 0.016 \pm 0.006$ for the $K^{+} K^{-}$ final state and $\phi_{s}=-0.057 \pm 0.060 \pm 0.011 \mathrm{rad}$ and $|\lambda|=1.01_{-0.06}^{+0.08} \pm 0.03$ for the $\pi^{+} \pi^{-}$final state. When combined together using also previous measurements at $7 \mathrm{TeV}$ and $8 \mathrm{TeV}$ [17, 18, 19, 20, 21] the final result is $\phi_{s}=-0.041 \pm 0.025 \mathrm{rad}$ and $|\lambda|=0.993 \pm 0.010$. This shows that $\phi_{s}$ is consistent with the Standard Model and is nearly $1.6 \sigma$ away from 0 , which indicates consistence with no $C P$ violation in interference between direct decay and after mixing. This is currently also the most precise measurement of $\phi_{s} .|\lambda|$ is consistent with no direct $C P$ violation.

\section{Conclusions}

The LHCb collaboration has recently reported measurements of a series of $C P$-violating parameters with an increased precision using data of $p p$ collisions at a centre-of-mass energy of $13 \mathrm{TeV}$. Among those, the first evidence of direct $C P$ violating in the charm sector represents an important milestone in particle physics. This comes nearly 20 years after the discovery of $C P$ violation in $B$ mesons by the Belle and BaBar collaborations in 2001. In the $B$ meson sector the updated measurement of the $\phi_{s}$ phase is the most precise to date and shows a $1.6 \sigma$ consistency with $C P$ conservation. The reported measurements have been done using Run 2 data and showed consistency with SM expectations. However, with the increase in statistics foreseen for Run 3 where $50 \mathrm{fb}^{-1}$ of data will be collected by 2028[22], sensitivity to BSM contributions is expected to increase further. 


\section{References}

[1] LHCb Collaboration, The LHCb Detector at the LHC, 10.1088/1748-0221/3/08/s08005

[2] R. Aaij et al., Performance of the LHCb Vertex Locator, Journal of Instrumentation, 9-09, 2014

[3] M. Adinolfi et al., Performance of the LHCb RICH Detector at LHC, arXiv:1211.6759

[4] M. Golden and B. Grinstein, Enhanced $C P$ violations in hadronic charm decays, Phys. Lett. B222 (1989) 501

[5] Y. Grossman, A. L. Kagan, and Y. Nir, New physics and $C P$ violation in singly Cabibbo suppressed D decays, Phys. Rev. D75 (2007) 036008

[6] LHCb Collaboration, R. Aaij et al., Observation of $C P$ Violation in Charm Decays, Phys. Rev. Lett. 122,211803

[7] LHCb collaboration, R. Aaij et al., Measurement of $C P$ asymmetry in $D^{0} \rightarrow K^{+} K^{-}$and $D^{0} \rightarrow \pi^{+} \pi^{-}$ decays, JHEP 07 (2014) 041

[8] LHCb collaboration, R. Aaij et al., Measurement of the difference of time-integrated $C P$ asymmetries in $D^{0} \rightarrow K^{+} K^{-}$and $D^{0} \rightarrow \pi^{+} \pi^{-}$decays, Phys. Rev. Lett. 116 (2016) 191601

[9] LHCb collaboration, R. Aaij et al., Search for $C P$ violation in $D_{s}^{+} \rightarrow K_{S}^{0} \pi^{+}, D^{+} \rightarrow K_{S}^{0} K^{+}, D^{+} \rightarrow \phi \pi^{+}$ decays, Phys. Rev. Lett.122(2019) 191803

[10] Updated results and plots available at http://ckmfitter.in2p3.fr/ and at http://www.utfit.org/

[11] LHCb collaboration, R. Aaij et al., Measurement of the $C P$-violating phase $\phi_{s}$ from $B_{s}^{0} \rightarrow J / \psi \pi^{+} \pi^{-}$ decays in $13 \mathrm{TeV}$ pp collisions, arXiv:1903.05530

[12] LHCb collaboration, R. Aaij et al., Updated measurement of time-dependent $C P$-violating observables in $B_{s}^{0} \rightarrow J / \psi K^{+} K^{-}$, arXiv: 1096.08356

[13] L. Zhang and S. Stone, Time-dependent Dalitz-plot formalism for $B_{q}^{0} \rightarrow J / \psi h^{+} h^{-}$, Phys. Lett. B719 (2013) 383

[14] LHCb collaboration, R. Aaij et al.,Measurement of resonant and $C P$ components in $B_{s}^{0} \rightarrow J / \psi \pi^{+} \pi^{-}$ decays, Phys. Rev.D89(2014) 092006,

[15] LHCb collaboration, R. Aaij et al., Tagged time-dependent angular analysis of $B_{s}^{0} \rightarrow J / \psi \phi$ decays at LHCb, LHCb-CONF-2012-002

[16] LHCb collaboration, R. Aaij et al., Measurement of the $C P$ asymmetry in $B_{s}^{0}$ and $\bar{B}_{s}^{0}$ mixing, Phys. Rev. Lett. 117 (2016) 061803

[17] LHCb collaboration, R. Aaij et al.,Precision measurement of $C P$ violation in $B_{s}^{0} \rightarrow J / \psi K^{+} K^{-}$decays, Phys. Rev. Lett.114(2015) 041801

[18] LHCb collaboration, R. Aaij et al.,Measurement of the $C P$-violating phase $\phi_{s}$ in $B_{s}^{0} \rightarrow J / \psi \pi^{+} \pi^{-}$ decays, Phys. Lett.B736(2014) 186

[19] LHCb collaboration, R. Aaij et al.,Measurement of the $C P$ violating phase and decay-width difference in $B_{s}^{0} \rightarrow \psi(2 S) \phi$ decays, Phys. Lett.B762(2016) 253

[20] LHCb collaboration, R. Aaij et al.,Measurement of the $C P$-violating phase $\phi_{s}$ in $\bar{B}_{s}^{0} \rightarrow D_{s}^{+} D_{s}^{-}$decays, Phys. Rev. Lett.113(2014) 211801

[21] LHCb collaboration, R. Aaij et al.,Resonances and $C P$-violation in $B_{s}^{0}$ and $\bar{B}_{s}^{0} \rightarrow J / \psi K^{+} K^{-}$decays in the mass region above the $\phi(1020)$, JHEP08(2017)893037

[22] Alessio Piucci, The LHCb Upgrade, 10.1088/1742-6596/878/1/012012 